\title{
A2A adenosine receptor antagonists protect the striatum against rotenone-induced neurotoxicity
}

\author{
Vincenzo Belcastro $^{\mathrm{a}, \mathrm{b}}$, Alessandro Tozzi ${ }^{\mathrm{a}, \mathrm{b}}$, Michela Tantucci ${ }^{\mathrm{a}, \mathrm{b}}$, Cinzia Costa ${ }^{\mathrm{a}, \mathrm{b}}$, Massimiliano Di Filippo ${ }^{\mathrm{a}, \mathrm{b}}$, \\ Alessia Autuori $^{\mathrm{a}, \mathrm{b}}$, Barbara Picconi ${ }^{\mathrm{b}}$, Sabrina Siliquini ${ }^{\mathrm{a}, \mathrm{b}}$, Elisa Luchetti ${ }^{\mathrm{a}, \mathrm{b}}$, \\ Franco Borsini ${ }^{c}$, Paolo Calabresi ${ }^{\text {a,b, }}$, \\ a Clinica Neurologia, Università di Perugia, Ospedale S. Maria della Misericordia, 06156 Perugia, Italy \\ b I.R.C.C.S., Fondazione Santa Lucia, 00143 Rome, Italy \\ c Sigma-tau Industrie Riunite, Via Pontina km 30,400 00040, Pomezia, Italy
}

\section{A R T I C L E I N F O}

\section{Article history:}

Received 7 October 2008

Revised 7 January 2009

Accepted 16 January 2009

Available online 29 January 2009

\section{Keywords:}

Adenosine

A2A receptor

Rotenone

Striatum

Neuroprotection

\begin{abstract}
A B S T R A C T
Adenosine $\mathrm{A} 2 \mathrm{~A}$ receptor has emerged as an attractive non-dopaminergic target in the experimental pharmacological therapy for Parkinson's disease (PD). Moreover, it has been postulated that A2A adenosine receptor antagonists exert neuroprotective effects in experimental models of PD and progressive supranuclear palsy (PSP). Interestingly, in both these pathological conditions a deficit of mitochondrial complex I has been found. Thus, utilizing extracellular and intracellular recordings from corticostriatal brain slices, we have tested the possible neuroprotective action of two A2A receptor antagonists, ST1535 and ZM241385, on the irreversible electrophysiological effects induced by the acute application of rotenone, a pesticide acting as a selective inhibitor of mitochondrial complex I activity. Both these antagonists reduced the rotenone-induced loss of corticostriatal field potential amplitude as well as the membrane depolarization caused by this toxin on striatal spiny neurons. The use of A2A receptor antagonists might represent a promising neuroprotective strategy in basal ganglia disorders involving a deficit of mitochondrial complex I activity.
\end{abstract}

(c) 2009 Elsevier Inc. All rights reserved.

\section{Introduction}

Dysfunction in mitochondrial energy metabolism and oxidative damage has been strongly implicated in the etiology of both Parkinson's disease (PD) and progressive supranuclear palsy (PSP) (Höglinger et al., 2005). Despite these neurodegenerative diseases present a distinctive pattern of neuronal cell loss and pathological hallmarks, mitochondrial deficits have been reported in tissues from both PD (Sulzer, 2007) and PSP patients (Burn and Lees, 2002). Experimental studies have reported that generalized complex I inhibition by the widely used natural pesticide rotenone causes the damage of both nigral and striatal neurons, in a pattern reminiscent of atypical parkinsonian syndromes such as PSP (Höglinger et al., 2005; Centonze et al., 2006).

Adenosine A2A receptor has emerged as an attractive nondopaminergic target in the pursuit of improved therapy for PD, based in part on its unique central nervous system (CNS) distribution. In particular, adenosine $\mathrm{A} 2 \mathrm{~A}$ receptors are abundant in the caudate-putamen, nucleus accumbens, and olfactory tubercle

\footnotetext{
* Corresponding author. Clinica Neurologica, Facoltà di Medicina e Chirurgia, Università degli Studi di Perugia, Ospedale S. Maria della Misericordia, 06156 Perugia, Italy. Fax: +390755784229.

E-mail address: calabre@unipg.it (P. Calabresi).
}

and can form functional heteromeric complexes with other Gprotein-coupled receptors, including dopamine (DA) D2, type 5 metabotropic glutamate (mGlu5) and adenosine A1 receptors (Schwarzschild et al., 2006). Interestingly, experimental findings have raised the possibility that $\mathrm{A} 2 \mathrm{~A}$ receptor antagonist-mediated neuroprotection extends beyond PD models of nigrostriatal neuron degeneration and can limit the damage to striatal output neurons induced by mitochondrial toxins (Alfinito et al., 2003; Schwarzschild et al., 2006; Chen et al., 2007).

The present study was aimed at investigating the possible neuroprotective role of A2A receptor antagonists against the neuronal damage triggered by rotenone. In particular, we utilized intracellular and extracellular electrophysiological recordings in an in vitro neurotoxic model obtained following the acute administration of a selective inhibitor of complex I of the mitochondrial respiratory chain.

\section{Materials and methods}

Preparation and maintenance of corticostriatal slices for electrophysiological recordings

Preparation and maintenance of rat corticostriatal slices have been previously described (Calabresi et al. 1997, 2001). Briefly, 
corticostriatal coronal slices (thickness, $270 \mu \mathrm{m}$ ) were cut from one to two-month-old male Wistar rats (Charles River Laboratories, Inc. Wilmington, MA, USA) using a vibratome. All the experiments were conducted in conformity with the European Communities Council Directive of November 1986 (86/609/ECC). A single slice was then transferred to a recording chamber and submerged in a continuously flowing Kreb's solution $\left(34^{\circ} \mathrm{C} ; 2.5-3 \mathrm{ml} / \mathrm{min}\right.$ ) bubbled with a $95 \% \mathrm{O}_{2}-5 \% \mathrm{CO}_{2}$ gas mixture. The composition of the solution was (in mM) $126 \mathrm{NaCl}, 2.5 \mathrm{KCl}, 1.2 \mathrm{MgCl}_{2}, 1.2 \mathrm{NaH}_{2} \mathrm{PO}_{4}, 2.4 \mathrm{CaCl}_{2}, 10$ glucose, and $25 \mathrm{NaHCO}_{3}$. Drugs were bath-applied by switching the solution to one containing known concentrations of drugs. Total replacement of the medium in the chamber occurred within $1 \mathrm{~min}$.

\section{Electrophysiology}

Extracellular recordings were obtained by using sharp microelectrodes pulled from borosilicate glass pipettes backfilled with $2 \mathrm{M} \mathrm{NaCl}$ (15-20 M $\Omega$ ). Intracellular recordings of striatal medium spiny neurons were obtained by using sharp microelectrodes pulled from borosilicate glass pipettes backfilled with $2 \mathrm{M} \mathrm{KCl} \mathrm{(30-}$ $60 \mathrm{M} \Omega$ ). An Axoclamp 2B amplifier (Axon Instruments, USA) was used for extracellular recordings. The field potential amplitude was defined as the average of the amplitude from the peak of the early positivity to the peak negativity and the amplitude from the peak negativity to peak late positivity. A glutamatergic corticostriatal synaptic excitatory post-synaptic potential (EPSP) or a field potential was evoked every $10 \mathrm{~s}$ by means of a bipolar electrode connected to a stimulator unit (Grass Telefactor, USA). The stimulating electrode was located in the white matter between the cortex and the striatum to activate corticostriatal fibers. The recording electrodes were invariably placed within the striatum. Quantitative data are expressed as a percentage of the field potential amplitudes or of the EPSP in respect to the relative control amplitude values, the latter representing the mean of responses recorded during a stable period (15 to $20 \mathrm{~min}$ ). Off-line analysis was performed using Clampfit (Axon Instruments, USA) and Microcal Origin (Northampton, MA, USA) softwares. KruskalWallis and Dunn's post-hoc test were used for statistical analysis. Values given in the figures and text are mean \pm standard error of the mean (SEM). The significance level was established at $p<0.05$ $(*)$ and $p<0.01(* *)$.

\section{Drugs}

Powders were dissolved in water or DMSO and then stored at $-20{ }^{\circ} \mathrm{C}$ in aliquots. Each aliquot was only used the day of experiment and then discarded. Drugs were applied by dissolving them to the desired final concentration in the external Kreb's solution. ZM241385 was from Tocris-Cookson (Bristol, UK); ST1535 was from Sigma-Tau (Pomezia, Italy); Rotenone was from Sigma-Aldrich (Milano, Italy); CGS21680 was from Tocris-Cookson (Bristol, UK).

\section{Results}

\section{Electrophysiological effects of rotenone}

In order to test whether inhibition of mitochondrial complex I by rotenone was able to alter the electrical neuronal activity in a corticostriatal brain slice preparation, field potentials were recorded from the striatum following the activation of glutamatergic corticostriatal inputs. As shown in the Figs. 1A, B, the application of rotenone for 30 min induced a progressive reduction of the field potential amplitude. After 30 min of application of this toxin the loss of the field potential amplitude was almost complete by utilizing a dose of $1 \mu \mathrm{M}$. After the wash-out of this toxin no recovery of the field potential was observed (Figs. 1B-G). This observation suggests that the utilized concentrations of rotenone induced irreversible electrophysiological alterations in striatal neurons reflecting neuronal death (Tozzi et al., 2007a; Costa et al., 2008).

The effect of rotenone was also investigated by utilizing intracellular recordings from 35 electrophysiologically identified principal spiny neurons. The main characteristics of these cells have been described in detail previously (Calabresi et al., 1997). These cells had high resting membrane potential $(-84 \pm 5 \mathrm{mV})$, low apparent input resistance $(39 \pm 9 \mathrm{M} \Omega)$ and action potentials of short duration $(1.1 \pm 0.3 \mathrm{~ms})$ and high amplitude $(102 \pm 4 \mathrm{mV})$. These cells were silent at rest and showed tonic firing activity during a long-duration depolarizing pulse. Rotenone, at the concentration of $1 \mu \mathrm{M}$ induced a slow membrane depolarization starting after 5$10 \mathrm{~min}$ from the onset of perfusion $(n=15$, Fig. $1 \mathrm{~F})$. The rotenoneinduced depolarization reached a plateau after $30 \mathrm{~min}$ from the onset of the application, when the membrane potential of the neuron was around $-20 \mathrm{mV}$ (Fig. 1F). Also in this case, the electrophysiological effect induced by the toxin was irreversible after its wash-out ( $n=5$, data not shown). It is worth to note that the rotenone-induced membrane depolarization was coupled to a reduction of the input resistance of the recorded cells ( $n=7$, data not shown).

$A 2 A$ receptors antagonism prevents the irreversible electrophysiological changes induced by the inhibition of mitochondrial complex I

A2A receptors are particularly abundant in the striatum while their expression is much lower in other brain areas (Schwarzschild et al., 2006). In the striatum, A2A receptors are selectively expressed by striatopallidal neurons, which also express dopamine D2 receptors; additionally, a proportion of $\mathrm{A} 2 \mathrm{~A}$ receptors are located presynaptically on corticostriatal terminals, where they control glutamate release. It is known that blockade of pre-synaptic A2A receptors reduces glutamate release in several brain regions. Thus, a reduction in glutamate release might be one mechanism by which the A2A receptor antagonists exert a neuroprotection (Chen et al., 2007). For this reason, we have investigated the possible neuroprotective effects of ST1535 and ZM241385, two A2A receptor antagonists, against the irreversible functional alterations caused by rotenone in striatal neurons. These antagonists were first preincubated for 10-15 min alone and then applied together with rotenone. As reported in the Figs. $1 \mathrm{~A}-\mathrm{C}, 1 \mu \mathrm{M}$ and $0.1 \mu \mathrm{M}$ ZM241385, and $10 \mu \mathrm{M}$ ST1535 significantly reduced the electrophysiological changes caused by rotenone $(n=8$ for each drug, $p<0.05) ; 1 \mu \mathrm{M}$ ST1535 produced a slight decrease of the field potential damage induced by rotenone, however, this effect was not significant $(n=8, p>0.05)$. The application of $10 \mu \mathrm{M}$ ST1535, $1 \mu \mathrm{M}$ and $0.1 \mu \mathrm{M}$ ZM241385 for $15-20 \mathrm{~min}$ did not alter per se the amplitude of the field potential (data not shown).

In order to further analyse the ability of ST1535 and ZM241385 to protect against the rotenone-induced neurotoxicity we also performed intracellular recordings from striatal spiny neurons. Interestingly, the preincubation of the slices with $10 \mu \mathrm{M}$ ST1535 $(n=10$, $p<0.01)$ or $0.1 \mu \mathrm{M}$ ZM241385 $(n=10, p<0.05)$ significantly delayed the onset of the rotenone-induced membrane depolarization (Fig. 1F). At these concentrations ST1535 and ZM241385 did not alter per se the membrane potential and the input resistance of the recorded neurons (data not shown).

Since the obtained data suggest that antagonism of A2A receptors exerts a neuroprotective role against rotenone-induced neurotoxicity, we also tested the hypothesis that the application of an agonist acting on these receptors could exert an additional detrimental effect. However, CGS21680 ( $3 \mu \mathrm{M}, n=8, p>0.05$ ), a selective A2A receptor agonist, did not alter the effects induced by $1 \mu \mathrm{M}$ rotenone (Fig. 1D). CGS21680 ( $1 \mu \mathrm{M}$ and $3 \mu \mathrm{M}, n=8$ for each 
A

control

in $\mathrm{ZM} 1 \mu \mathrm{M}$

in ST $10 \mu \mathrm{M}$

pre-rotenone 30' rotenone pre-rotenone 30' rotenone pre-rotenone 30' rotenone

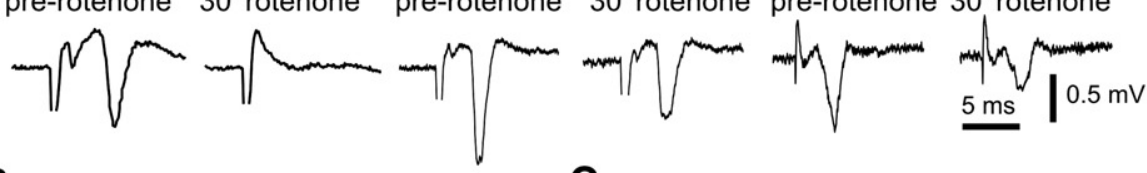

B

C
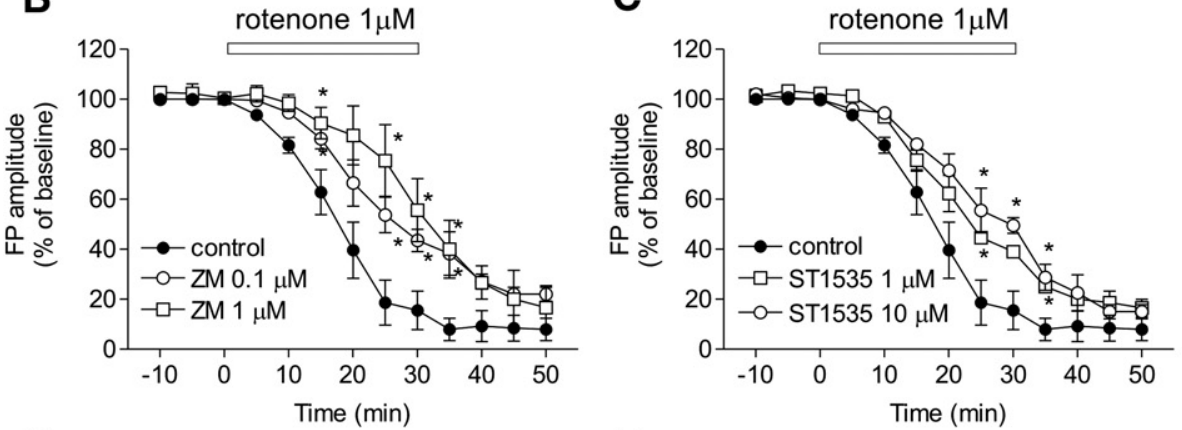

D

E

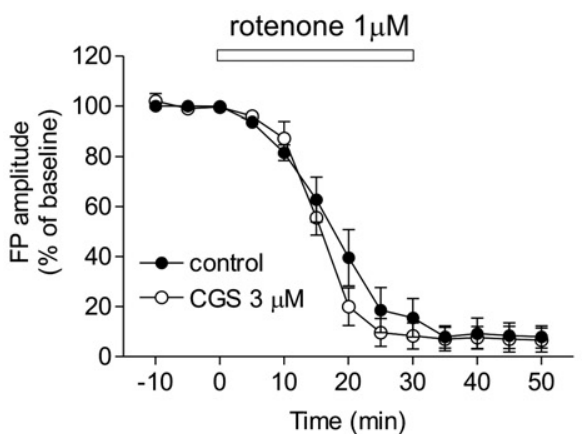

$\mathbf{F}$
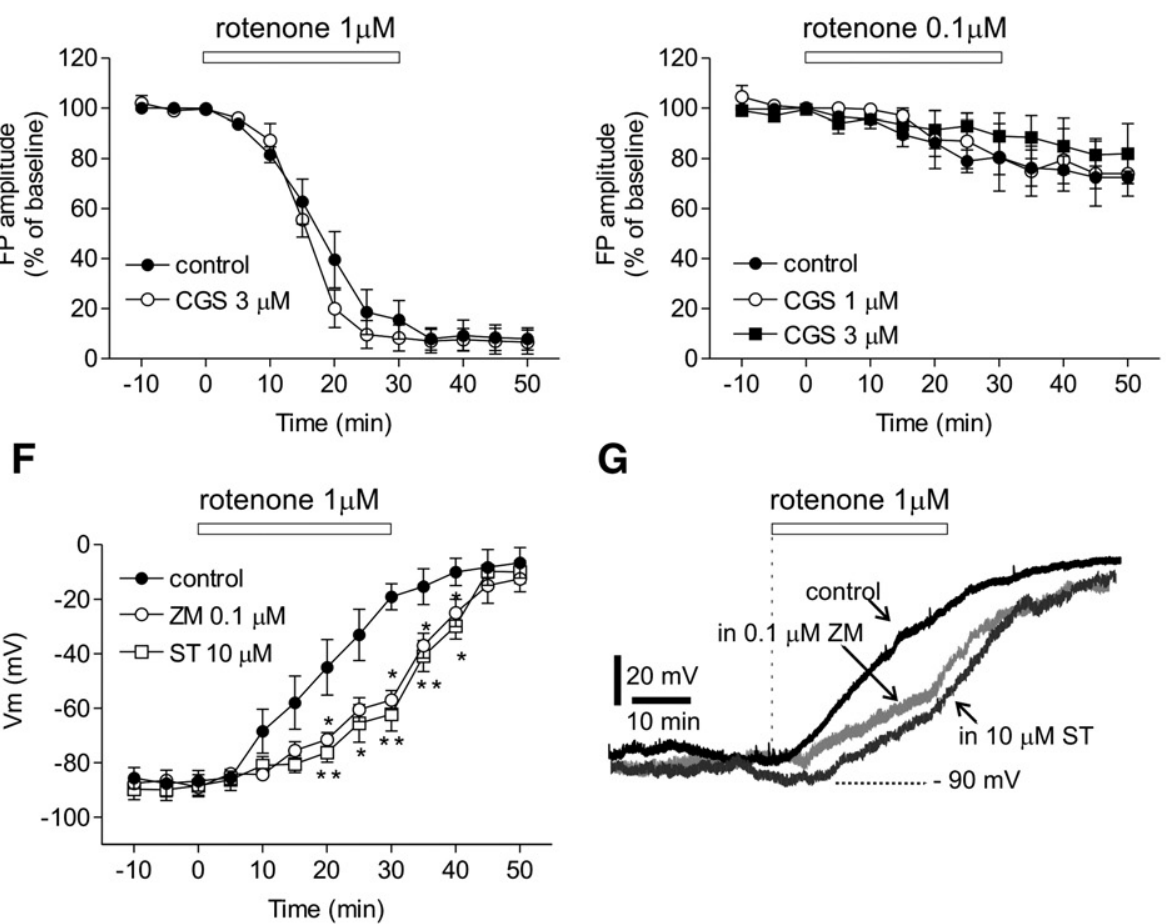

G

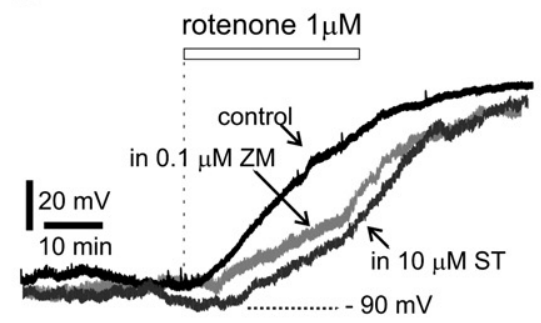

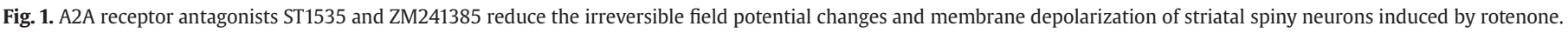

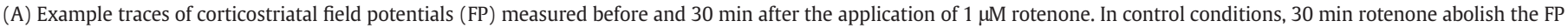

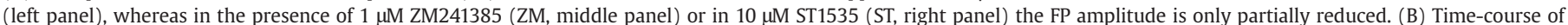

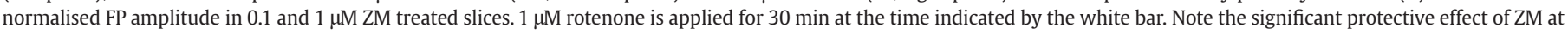

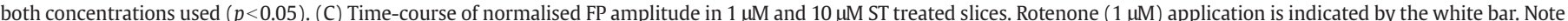

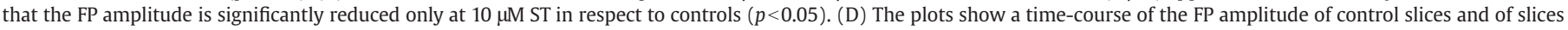

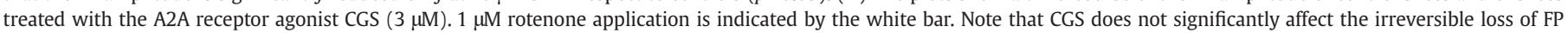

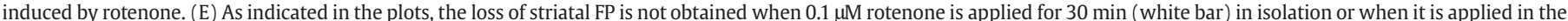

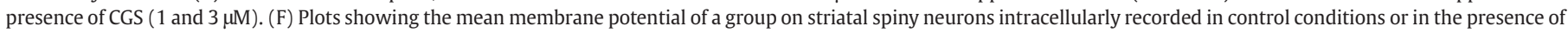

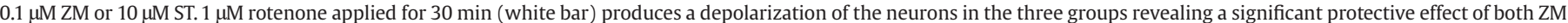

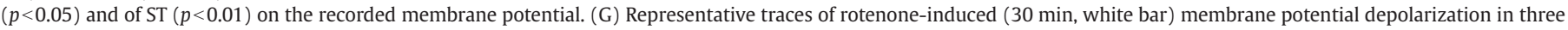
striatal spiny neurons recorded in control conditions, in the presence of $0.1 \mu \mathrm{M}$ ZM or in $10 \mu \mathrm{M}$ ST.

concentration, $p>0.05)$ also failed to enhance the electrophysiological effects induced by a lower dose $(0.1 \mu \mathrm{M})$ of rotenone (Fig. 1E).

\section{Discussion}

The major finding of the present study is that in corticostriatal slices $\mathrm{A} 2 \mathrm{~A}$ receptor antagonism reduces the irreversible functional alterations caused by rotenone in striatal neurons.
Chronic rotenone treatment inhibits the mitochondrial chain complex I and damages both nigral and striatal neurons in two experimental models of akinetic syndromes such as PD (Betarbet et al., 2000) and PSP (Höglinger et al., 2005). Rotenone toxicity is thus dependent upon oxidative stress, a primary mechanism of toxicity (Sherer et al., 2007). Glutamate-mediated excitotoxicity is believed to substantially contribute to neuronal death during degenerative processes, and evidence exists that, at least under certain circumstances, 
mitochondrial impairment may sensitize neurons to glutamate NMDA receptor-mediated excitotoxicity and vice versa (Greene and Greenamyre 1996; Luetjens et al., 2000; Calabresi et al., 2001). One of the possible explanations for the cell-type specific vulnerability induced by rotenone in the basal ganglia is the dopamine-dependence of the rotenone-induced neurodegeneration. In fact, it is possible that the high endogenous dopamine levels present both in the pars compacta of the substantia nigra and in the striatum render these structures selectively prone to toxicity induced by mitochondrial complex I inhibition (Costa et al., 2008). Accordingly, a close link between dopamine transmission and mitochondrial dysfunction has been hypothesized in the pathogenesis of neurodegenerative disorders of the basal ganglia, such as PD and PSP (Sulzer, 2007).

In the last few years, adenosine $\mathrm{A} 2 \mathrm{~A}$ receptor antagonists have been consistently reported to exert antiparkinsonian and neuroprotective effects in different experimental models of neurodegeneration, including PD (Schwarzschild et al., 2006). To the best of our knowledge this is the first study aimed at investigating the possible neuroprotective effect of $\mathrm{A} 2 \mathrm{~A}$ receptors antagonism against the irreversible functional alterations caused by rotenone in striatal spiny neurons.

The lack of effect of the selective A2A receptor agonist CGS21680 in enhancing the detrimental action of rotenone on striatal neurons could be explained postulating that, at least during metabolic impairment, $\mathrm{A} 2 \mathrm{~A}$ receptors are already saturated by endogenous adenosine.

Although the mechanisms responsible for the neuroprotective effects of adenosine A2A receptor antagonists are very complex, a normalisation of abnormally elevated glutamate levels might play a pivotal role (Ferre et al., 2005). Accordingly, our recent findings support the possibility that endogenous adenosine may activate A2A receptors located in presynaptic corticostriatal terminals, thereby antagonizing D2 receptors and reducing DA-mediated inhibition of glutamate release (Tozzi et al., 2007b). In fact, co-application of quinpirole and ST1535 or ZM241385 significantly reduced the amplitude of excitatory synaptic potentials. This inhibitory effect was associated with an increased paired-pulse facilitation suggesting a presynaptic mechanism of action. Accordingly, whole-cell recordings showed that the concomitant activation of D2 receptors and the antagonism of A2A receptors decreased the frequency of spontaneous excitatory currents without affecting their amplitude. These results suggest that $\mathrm{A} 2 \mathrm{~A}$ and $\mathrm{D} 2$ receptors converge in the control of corticostriatal glutamatergic transmission by exerting an opposite function. Thus, it is conceivable that the reduced glutamate release might be one of the mechanisms underlying the neuroprotective effect of A2A receptor antagonists. An alternative mechanism has been recently postulated for other neuroprotective agents able to reduce striatal rotenone-induced electrophysiological changes (Costa et al., 2008). In particular, we found that carbamazepine, unlike other tested antiepileptic drugs, exerts a potent neuroprotective action against rotenone-induced striatal neuronal dysfunction by a mechanisms requiring the activation of $G_{A B A_{A}}$ receptors via endogenous GABA. Since differential targeting of GABAergic transmission may represent a possible therapeutic strategy against basal ganglia neurodegenerative disorders involving mitochondrial complex I dysfunction, it is possible that A2A antagonists might exert their neuroprotective effects through the modulation of the GABAergic system.

Future studies, analysing in detail the effects of rotenone on both spontaneous and evoked excitatory and inhibitory striatal synaptic transmission by whole-cell patch clamp studies, will help to elucidate the possible contribution of glutamatergic and/or GABAer- gic systems to the pharmacological effects of A2A receptor antagonists.

In conclusion, our findings further support that A2A receptor antagonists might represent promising neuroprotective agents against the toxicity induced by mitochondrial complex inhibition, however their true therapeutic potential needs to be confirmed in a wider range of doses and in in vivo models of neurodegenerative disorders.

\section{Acknowledgments}

We wish to thank Mr. C. Spaccatini for his excellent technical assistance. This study was supported by the following grants: Progetti Finalizzati e Strategici Ministero della Salute 2005/2006/2007 (P.C., B. P.), Fondazione Cassa di Risparmio Perugia (P.C.), HEALTH-2007-22918, REPLACES (P.C.) and a Sigma-Tau grant (P.C.).

\section{References}

Alfinito, P.D., Wang, S.P., Manzino, L., Rijhsinghani, S., Zeevalk, G.D., Sonsalla, P.K., 2003 Adenosinergic protection of dopaminergic and GABAergic neurons against mitochondrial inhibition through receptors located in the substantia nigra and striatum, respectively. J. Neurosci. 23, 10982-10987.

Betarbet, R., Sherer, T.B., MacKenzie, G., Garcia-Osuna, M., Panov, A.V., Greenamyre, J.T. 2000. Chronic systemic pesticide exposure reproduces features of Parkinson's disease. Nat. Neurosci. 3, 1301-1306.

Burn, D.J., Lees, A.J., 2002. Progressive supranuclear palsy: where are we now? Lancet Neurol. 1, 359-369.

Calabresi, P., Ascone, C.M., Centonze, D., Pisani, A., Sancesario, G., D'Angelo, V., Bernardi, G., 1997. Opposite membrane potential changes induced by glucose deprivation in striatal spiny neurons and in large aspiny interneurons. J. Neurosci. 17, 1940-1949.

Calabresi, P., Gubellini, P., Picconi, B., Centonze, D., Pisani, A., Bonsi, P., Greengard, P., Hipskind, R.A., Borrelli, E., Bernardi, G., 2001. Inhibition of mitochondrial complex I induces a long-term potentiation of NMDA-mediated synaptic excitation in the striatum requiring endogenous dopamine. J. Neurosci. 21, 5110-5120.

Centonze, D., Prosperetti, C., Barone, I., Rossi, S., Picconi, B., Tscherter, A., De Chiara, V. Bernardi, G., Calabresi, P., 2006. NR2B-containing NMDA receptors promote the neurotoxic effects of 3-nitropropionic acid but not of rotenone in the striatum. Exp. Neurol. 202, 470-479.

Costa, C., Belcastro, V., Tozzi, A., Di Filippo, M., Tantucci, M., Siliquini, S., Autuori, A. Picconi, B., Spillantini, M.G., Fedele, E., Pittaluga, A., Reiteri, M., Calabresi, P., 2008. Electrophysiology and pharmacology of striatal neuronal dysfunction induced by mitochondrial complex I inhibition. J. Neurosci. 28, 8040-8052.

Chen, J., Sonsalla, P., Pedata, F., Melani, A., Domenici, M., Popoli, P., Geiger, J., Lopes, L., Mendonc, A., 2007. Adenosine A2A receptors and brain injury: broad spectrum of neuroprotection, multifaceted actions and "fine tuning" modulation. Progr. Neurobiol. 83, 310-331.

Ferre, S., Borycz, J., Goldberg, S.R., Hope, B.T., Morales, M., Lluis, C., Franco, R., Ciruela, F. Cunha, R., 2005. Role of adenosine in the control of homosynaptic plasticity in striatal excitatory synapses. J. Integr. Neurosci. 4, 445-464.

Greene, J.G., Greenamyre, J.T., 1996. Bioenergetics and glutamate excitotoxicity. Prog. Neurobiol. 48, 613-634.

Höglinger, G.U., Lannuzel, A., Khondiker, M.E., Michel, P.P., Duyckaerts, C., Féger, J. Champy, P., Prigent, A., Medja, F., Lombes, A., Oertel, W.H., Ruberg, M., Hirsch, E.C., 2005. The mitochondrial complex I inhibitor rotenone triggers a cerebral tauopathy. J. Neurochem. 95, 930-939.

Luetjens, C.M., Bui, N.T., Sengpiel, B., Munstermann, G., Poppe, M., Krohn, A.J., Bauerbach, E., Krieglstein, J., Prehn, J.H., 2000. Delayed mitochondrial dysfunction in excitotoxic neuron death: cytochrome $c$ release and a secondary increase in superoxide production. J. Neurosci. 200, 5715-5723.

Sherer, T.B., Richardson, J.R., Testa, C.M., Seo, B.B., Panov, A.V., Yagi, T., Matsuno-Yagi, A. Miller, G.W., Greenamyre, J.T., 2007. Mechanism of toxicity of pesticides acting at complex I: relevance to environmental etiologies of Parkinson's disease. J. Neurochem. 100, 1469-1479.

Schwarzschild, M., Agnati, L., Fuxe, K., Chen, J.F., Morelli, M., 2006. Targeting adenosine A2A receptors in Parkinson's disease. Trends Neurosci. 29, 647-654.

Sulzer, 2007. Multiple hit hypotheses for dopamine neuron loss in Parkinson's disease. Trends Neurosci. 5, 244-250.

Tozzi, A., Costa, C., Di Filippo, M., Tantucci, M., Siliquini, S., Belcastro, V., Parnetti, L., Picconi, B., Calabresi, P., 2007a. Memantine reduces neuronal dysfunctions triggered by in vitro ischemia and 3-nitropropionic acid. Exp. Neurol. 207, 218-226.

Tozzi, A., Tscherter, A., Belcastro, V., Tantucci, M., Costa, C., Picconi, B., Centonze, D. Calabresi, P., Borsini, F., 2007b. Interaction of A2A adenosine and D2 dopamine receptors modulates corticostriatal glutamatergic transmission. Neuropharmacology $53,783-789$. 